\title{
Studies on the Safety and Efficacy of Pyrotinib in the Treatment of HER2- Positive Advanced Solid Tumors Excluding Breast Cancer
}

This article was published in the following Dove Press journal: Cancer Management and Research

\author{
Yuzhen Yin' \\ Hui Yang (D) ${ }^{2}$ \\ Zhuo $\mathrm{Liu}^{3}$ \\ Jie $\operatorname{Tan}^{2}$ \\ Chunrong Zhu ${ }^{4}$ \\ Minbin Chen ${ }^{5}$ \\ Rengui Zhou ${ }^{6}$ \\ Lei $\mathrm{Wang}^{7}$ \\ Jun Qian (iD ${ }^{2,8}$
}

'Department of Tumor Center, The Affiliated Jiangsu Shengze Hospital of Nanjing Medical University, Suzhou, Jiangsu, People's Republic of China; ${ }^{2}$ Department of Oncology, Suzhou Municipal Hospital, Suzhou, Jiangsu, People's Republic of China; ' ${ }^{3}$ Department of Oncology, Zhangjiagang First People's Hospital,

Zhangjiagang, Jiangsu, People's Republic of China; ${ }^{4}$ Department of Oncology, The First Affiliated Hospital of Soochow University, Suzhou, Jiangsu, People's Republic of China; ${ }^{5}$ Department of Oncology, The First People's Hospital of Kunshan, Kunshan, Jiangsu, People's Republic of China; ${ }^{6}$ Department of Oncology, The 904th Hospital of the Joint Logistics Support Force of the Chinese People's Liberation Army, Wuxi, Jiangsu, People's Republic of China; ${ }^{7}$ Department of Breast Surgery, The First People's Hospital of Changzhou, Changzhou, Jiangsu, People's Republic of China; ${ }^{8}$ Department of Oncology, Jiangsu Province Hospital of Chinese Medicine, Affiliated Hospital of Nanjing University of Chinese Medicine, Nanjing, Jiangsu, People's Republic of China

Correspondence: Jun Qian

Department of Oncology, Jiangsu Province Hospital of Chinese Medicine, Affiliated Hospital of Nanjing University of Chinese Medicine, 155 Hanzhong Road, Qinhuai District, Nanjing, Jiangsu, People's Republic of China

Email junqian04I5@I26.com

Lei Wang

Department of Breast Surgery, The First People's Hospital of Changzhou, No. 185, Juqian Street, Changzhou, Jiangsu, People's Republic of China

Email 592722409@qq.com
Background: Human epidermal growth factor receptor 2 (HER2) is a member of the ErbB family and is a key proto-oncogene in solid tumors. This pilot study investigated the safety and efficacy of pyrotinib in HER2-positive non-breast advanced solid tumors.

Patients and Methods: Twenty-five patients with HER2-positive advanced solid tumors excluding breast cancer were enrolled to receive pyrotinib-based therapy. The primary end point was progression-free survival (PFS).

Results: The median PFS and overall survival (OS) were 3.5 months (95\% CI: $2.2-5.0$ months) and 9.6 months (95\% CI: 4.4-9.9 months), respectively. Ten patients with lung cancer and 9 patients with gastric cancer had a median PFS of 2.5 months $(95 \% \mathrm{CI}$ : 0.97-6.53 months) and 2.9 months (95\% CI: 1.50-7.17 months), respectively. The median OS was 9.9 months (95\% CI: 4.4-9.9 months) in patients with lung cancer and 5.9 months (95\% CI: 4.0-9.6 months) in patients with gastric cancer. No statistical significance of a median OS was observed, nonetheless, patients receiving $>3$ lines had a numerically lower median OS than those receiving $\leq 3$ lines of treatment ( 9.9 vs 5.1 months, $P=0.706$ ). All 23 patients were available for efficacy evaluation. The objective response rate (ORR) was $52.17 \%$ and disease control rate (DCR) was $91.3 \%$. The ORR for lung cancer was $44.4 \%$ and for gastric cancer was 50\%. In addition, the DCR for lung cancer was $77.8 \%$ and for stomach cancer was $100 \%$. Moreover, patients receiving $\leq 3$ lines of treatment had a numerically higher DCR than those receiving $>3$ lines of treatment $(94.1 \%$ vs $83.3 \%, \mathrm{P}=0.462)$. The most common treatment-related adverse events (TRAEs) were diarrhea (92\%), but only 5 (20\%) patients reported grade 3 diarrhea which could be well controlled.

Conclusion: Pyrotinib-based therapy demonstrates promising efficacy for HER2-positive advanced solid tumors excluding breast cancer and toxicities could be well controlled. The study is a pilot study motivating larger studies to elucidate the safety and efficacy of pyrotinib in non-breast solid tumors.

Keywords: pyrotinib, HER2-positive, solid tumor

\section{Introduction}

Human epidermal growth factor receptor 2 (HER2) is a member of the ErbB family and is a key proto-oncogene in solid tumors. HER2-positive is a key oncogenic driver event for approximately $15-20 \%$ of breast cancers, with pathogenesis mainly being the continuous expression of HER2 and the activation of its downstream signaling pathway. ${ }^{1,2}$ Preclinical studies have shown that the $P I 3 K / m T O R$ pathway plays a vital role in the HER2 downstream cascade. Over the past two decades, HER2-targeted therapies have improved the outcomes in patients with early and 
advanced HER2-positive breast cancer. ${ }^{3-5} H E R 2$-positive has also been described in a variety of other solid tumors including colon adenocarcinoma, squamous cell carcinoma of lung, intrahepatic cholangiocarcinoma, amongst others, and has been considered to be a vital prognostic and predictive marker for tumor. ${ }^{6,7}$ Although HER2-targeting drugs (trastuzumab, pertuzumab, adotrastuzumab emtansine [TDM1], lapatinib) have improved survival in patients with HER2-positive lung, stomach, and cervical cancers, both inherent and developed resistance to HER2-targeting drugs still limit our effective management of this highly aggressive cancer subtype. ${ }^{8-10}$ Therefore, effective HER2-targeted therapies for these patients are an unmet need, and efforts are being made to develop new anti-HER2 drugs.

Pyrotinib is a newer oral, irreversible, tyrosine kinase inhibitor (TKI) of three members of the HER family (HER1, HER2, and HER4). ${ }^{11}$ A previous study demonstrated effective proliferation inhibition of HER2-positive cells both in vivo and in vitro. ${ }^{11,12}$ It was approved in China for HER2-positive advanced breast cancer in August 2018 because of the promising result of the Phase II study. ${ }^{13}$ Recently, PHOEBE, a randomized Phase III trial, showed that pyrotinib plus capecitabine had significantly longer median PFS (12.5 months vs 6.8 months, $P<0.0001)$ and higher objective response rate (ORR) $(67.2 \%$ vs $51.5 \%, P=0.0091)$ than lapatinib plus capecitabine in patients with HER2-positive metastatic breast cancer, and prolonged the median progression-free survival (PFS) by 5.7 months. ${ }^{14}$ Multiple clinical studies have confirmed the promising efficacy of pyrotinib in breast cancer, so this study investigated the safety and efficacy of pyrotinib in HER2-positive non-breast advanced solid tumors.

\section{Patients and Methods}

\section{Study Design, Treatment, and Assessment} This was an observational, multicenter and retrospective study that enrolled 25 patients from 14 sites from September 2018 to May 2019. All patients received pyrotinib-based therapy for a 21-day cycle until unacceptable toxicity or disease progression. Tumor imaging assessments were performed every 2 or 3 cycles according to Response Evaluation Criteria in Solid Tumors (RECIST), version 1.1. Written informed consents were obtained from all patients included in the study, and this study was approved by Suzhou Municipal Hospital Review
Committee. The study was conducted in accordance with the Declaration of Helsinki.

\section{Patient Population}

Patients were eligible if they (i) had a diagnosis of advanced HER2-positive (IHC $3+$, or IHC $2+$ confirmed by FISH) solid tumor except breast cancer, (ii) were 18-75 years old, (iii) had a predicted life expectancy of $\geq 3$ months, (iv) had adequate kidney, hematologic, and liver function. Tumor tissues are classified as HER2-positive if they are scored as $3+$ by an IHC method defined as uniform membrane staining for $H E R 2$ in $10 \%$ or more of tumor cells (Figure 1). ${ }^{15}$

Patients were excluded if they had received surgery within 4 weeks before enrollment, or if they had received prior treatment with pyrotinib. Other exclusive criteria included: a left ventricular ejection fraction of $<50 \%$; missing information for treatment; less than one cycle of pyrotinib-based therapy. No restriction on prior therapy was required.

\section{Study End Points and Assessments}

The primary outcome was progression-free survival. We calculated PFS as the time from pyrotinib initiation to disease progression or death as a result of any cause. The secondary outcome included overall survival (OS), objective response rate (ORR), and disease control rate (DCR). OS was calculated from date of pyrotinib initiation to death. ORR and DCR were evaluated according to RECIST v1.1. Other efficacy endpoints included toxicities assessed in accordance with the National Cancer Institute

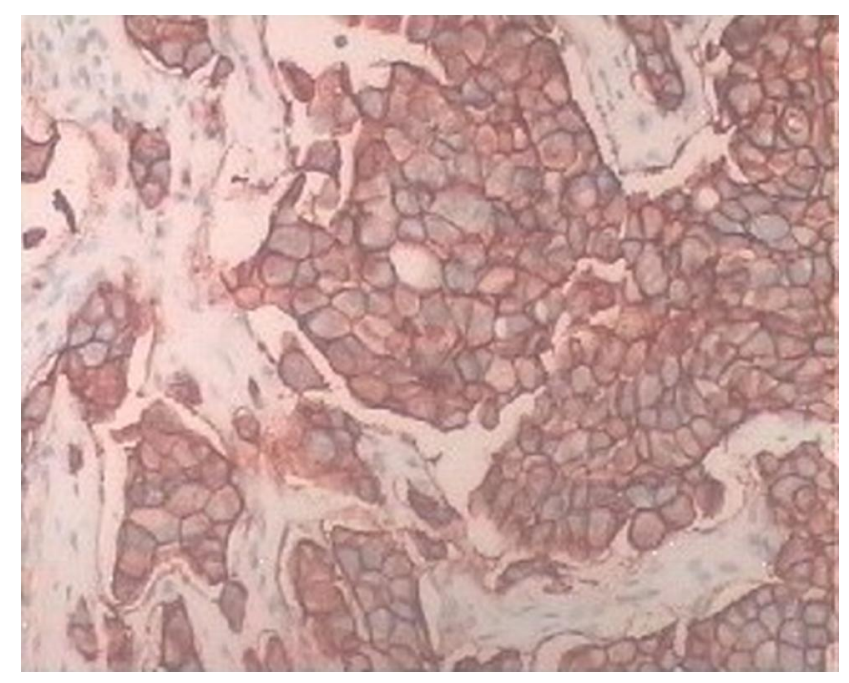

Figure I Representative image of HER2-positive tumor tissue by IHC staining. 
Common Terminology Criteria for Adverse Events (NCI CTCAE), version 5.0.

\section{Statistical Analyses}

All descriptive statistics are presented as median (range) or number of patients (percentage). Survival estimates, including OS and PFS, was estimated using the Kaplan-Meier product-limit and compared by logrank test. The $95 \%$ confidence interval (CI) for ORR and DCR were calculated using the Clopper-Pearson method. All statistical tests were considered statistically significant at $P<0.05$. Statistical analyses were performed using SPSS version 23.0.

\section{Results}

\section{Patient Characteristics}

Overall, 25 patients across 14 sites who had received pyrotinib-based therapy between September 2018 and May 2019 for HER2-positive advanced solid tumors excluding breast cancer were included. All the patients received at least one dose of pyrotinib-based therapy and were included in the safety analysis. Among them, 23 patients were available for efficacy evaluation.

Patient characteristics are shown in Table 1. Median age was 64 (range 37-73 years). All 25 patients had HER2-positive solid tumor, including 10 lung cancers, 9 gastric cancers, and 6 other cancers; $72 \%$ of the patients were male and the median number of lines of therapy was 3. Immunohistochemistry (IHC) was used for assessing HER-2 status in 14 (56\%) patients. Fluorescence in situ hybridization (FISH) was used for 11 (44\%) patients. Overall, 25 patients had HER2-positive tumors as assessed by IHC and FISH. Nineteen (76.\%) patients were previously administered anti-HER2 drugs. Among them, 15 $(60 \%)$ patients had received a single type of anti-HER2 drug (trastuzumab); 4 (16\%) patients had received trastuzumab and lapatinib. Twenty (80\%) patients received pyrotinib-based therapy as a third or further line treatment. Treatment regimens were pyrotinib plus capecitabine (11/ $25)$, pyrotinib combined with other chemotherapy drugs (6/25), pyrotinib monotherapy (7/25), pyrotinib combined with trastuzumab (1/25), and pyrotinib combined with bevacizumab (1/25). Twenty-one (84\%) patients initiated pyrotinib treatment at $400 \mathrm{mg}, 2(8 \%)$ patients started with $320 \mathrm{mg}$, and $2(8 \%)$ patients had a starting dose of $160 \mathrm{mg}$.

\section{Efficacy}

The 23 patients available for efficacy evaluation included 9 with lung cancers, 8 with gastric cancers, and 6 with other cancers. Twelve (52.17\%) patients had partial response (PR), 9 (39.13\%) patients achieved stable disease (SD), and $2(8.70 \%)$ patients had progression of disease (PD), resulting in an ORR of $52.17 \%$ and DCR of $91.3 \%$. The details of each patient's best response are listed in Table 2. Subgroup analysis was based on types of tumors including lung cancer and stomach cancer. The ORR and DCR for pyrotinib-based therapy in different kinds of tumors are shown in Table 3. The ORR for lung cancer was $44.4 \%$ and for stomach cancer was $50 \%$. In addition, the DCR for lung cancer was $77.8 \%$ and for stomach cancer was $100 \%$. In addition, patients receiving $\leq 3$ lines of treatment had a numerically higher DCR than those receiving $>3$ lines of treatment $(94.1 \%$ vs 83.3\%, $P=0.462$ ) (Table 4).

The median PFS and OS were 3.5 months $(95 \% \mathrm{CI}$ : 2.2-5.0 months) (Figure 2) and 9.6 months (95\% CI: 4.4-9.9 months) (Figure 3), respectively. Ten patients with lung cancer and 9 patients with gastric cancer had a median PFS of 2.5 months (95\% CI: 0.97-6.53 months) and 2.9 months (95\% CI: $1.50-7.17$ months), respectively (Figure 4). The median OS was 9.9 months (95\% CI: $4.4-9.9$ months) in patients with lung cancer and 5.9 months $(95 \%$ CI: 4.0-9.6 months) in patients with gastric cancer (Figure 5). No statistical significance of a median OS was observed, nonetheless, patients receiving $>3$ lines had a numerically lower median OS than those receiving $\leq 3$ lines of treatment (9.9 vs 5.1 months, $P=0.706$ ) (Figure 6 ).

\section{Safety}

All $25(100 \%)$ patients experienced at least one treatmentrelated adverse event (TRAE), and most of these were grade 1 or 2 . No grade 4 or 5 TRAEs occurred. The most common TRAEs were diarrhea (92\%), but only 5 (20\%) patientsreported grade 3 diarrhea which could be well controlled. Other AEs included asthenia (32\%), handfoot syndrome (24\%), vomiting (8\%), stomatitis (4\%), and anemia (4\%), and all were grade 1 or 2 . There were no deaths related to pyrotinib (Table 5).

\section{Discussion}

For many cancers, such as lung cancer, gastric cancer, and colorectal cancer, molecular profiling-guided therapy has been applied in routine clinical practice. ${ }^{16-19}$ However, for 
Table I Patient Characteristics

\begin{tabular}{|c|c|}
\hline Characteristic & $\mathbf{N}=\mathbf{2 5}, \mathrm{n}(\%)$ \\
\hline Median age, years (range) & $64(37-73)$ \\
\hline \multicolumn{2}{|l|}{ Sex } \\
\hline Male & $18(72.00)$ \\
\hline Female & $7(28.00)$ \\
\hline \multicolumn{2}{|l|}{ ECOG performance status } \\
\hline $0-1$ & $9(36.00)$ \\
\hline$\geq 2$ & $16(64.00)$ \\
\hline \multicolumn{2}{|l|}{ Tumor Type } \\
\hline Lung & $10(40.00)$ \\
\hline Gastric & $9(36.00)$ \\
\hline Colorectal & $2(8.00)$ \\
\hline Thymus & $2(8.00)$ \\
\hline Ovarian & I (4.00) \\
\hline Gallbladder & I (4.00) \\
\hline \multicolumn{2}{|l|}{ HER-2 status } \\
\hline IHC 3+ & $14(56.00)$ \\
\hline $\mathrm{FISH}+$ & II (44.00) \\
\hline \multicolumn{2}{|l|}{ Previous anti-HER2 antibody treatment } \\
\hline Trastuzumab & $15(60.00)$ \\
\hline Trastuzumab+ lapatinib & $4(16.00)$ \\
\hline \multicolumn{2}{|l|}{ Treatment stage } \\
\hline I & $3(12.00)$ \\
\hline 2 & $2(8.00)$ \\
\hline 3 & $14(56.00)$ \\
\hline$>3$ & $6(24.00)$ \\
\hline \multicolumn{2}{|l|}{ Treatment regimen } \\
\hline Pyrotinib + Capecitabine & II (44.00) \\
\hline Pyrotinib & $7(28.00)$ \\
\hline Pyrotinib + Paclitaxel (Albumin Bound) & $2(8.00)$ \\
\hline Pyrotinib + Irinotecan & I (4.00) \\
\hline Pyrotinib + Pemetrexed & I (4.00) \\
\hline Pyrotinib + Osimertinib & I (4.00) \\
\hline Pyrotinib + Bevacizumab & I (4.00) \\
\hline Pyrotinib + Trastuzumab & I (4.00) \\
\hline \multicolumn{2}{|l|}{ Starting dosage of Pyrotinib } \\
\hline $400 \mathrm{mg}$ & $21(84.00)$ \\
\hline $320 \mathrm{mg}$ & $2(8.00)$ \\
\hline $160 \mathrm{mg}$ & $2(8.00)$ \\
\hline
\end{tabular}

many kinds of malignant tumors, the incidence of targetable molecular alterations is very low, which makes the development and research of tumor-targeted drugs challenging. One
Table 2 Tumor Response

\begin{tabular}{|l|l|}
\hline Best Response & $\mathbf{N}=\mathbf{2 3}, \mathbf{n}$ (\%) \\
\hline PR & $12(52.17)$ \\
SD & $9(39.13)$ \\
PD & $2(8.70)$ \\
ORR & $12(52.17)$ \\
DCR & $21(91.3)$ \\
\hline
\end{tabular}

Table 3 Tumor Response in Lung Cancer and Gastric Cancer

\begin{tabular}{|l|l|l|}
\hline Best Response & Lung Cancer & Gastric Cancer \\
\hline PR, n & 4 & 4 \\
SD, n & 3 & 4 \\
PD, n & 2 & - \\
ORR, n (\%) & $44.4 \%$ & $50 \%$ \\
DCR, n (\%) & $77.8 \%$ & $100 \%$ \\
\hline
\end{tabular}

way is to use "basket trials" to confirm that the presence of a biomarker is more appropriate for the tumor type. ${ }^{20-22}$ The uniqueness of this trial is that it can systematically use centralized analysis to explore many treatment options from patients at 14 clinical sites based on a master protocol. This report involves evaluating pyrotinib for the treatment of HER2-positive advanced solid tumors excluding breast cancer and is a vital complement to the existing data on pyrotinib.

In our study, the most intriguing result of this trial was an ORR of $52.17 \%$, numerically higher than that of adotrastuzumab emtansine (TDM1) reported in patients with tumors excluding breast and gastric/gastroesophageal junction (GEJ) adenocarcinomas $(5.6 \%),{ }^{23}$ and close to the ORR result of trastuzumab in patients with advanced solid tumor malignancies of $63 \% .^{24}$ Notably, PR was reported in $44.4 \%$ (4/9) lung cancers and 50\% (4/8) gastric cancers in our study. This warrants further study of pyrotinib in lung and gastric cancers in larger dedicated trials. Moreover, patients receiving $\leq 3$ lines of treatment had a higher DCR than those receiving $>3$ lines of treatment. Pyrotinib is highly beneficial to third-or-lower-line

Table 4 Tumor Response in Patients Receiving $\leq 3$ Lines and $>3$ Lines Treatment

\begin{tabular}{|l|l|l|}
\hline Best Response & $\leq \mathbf{3}$ Lines & >3 Lines \\
\hline PR, n & 10 & 2 \\
SD, $\mathrm{n}$ & 6 & 3 \\
PD, $\mathrm{n}$ & 1 & 1 \\
ORR, $\mathrm{n}(\%)$ & $58.9 \%$ & $33.3 \%$ \\
DCR, $\mathrm{n}(\%)$ & $94.1 \%$ & $83.3 \%$ \\
\hline
\end{tabular}




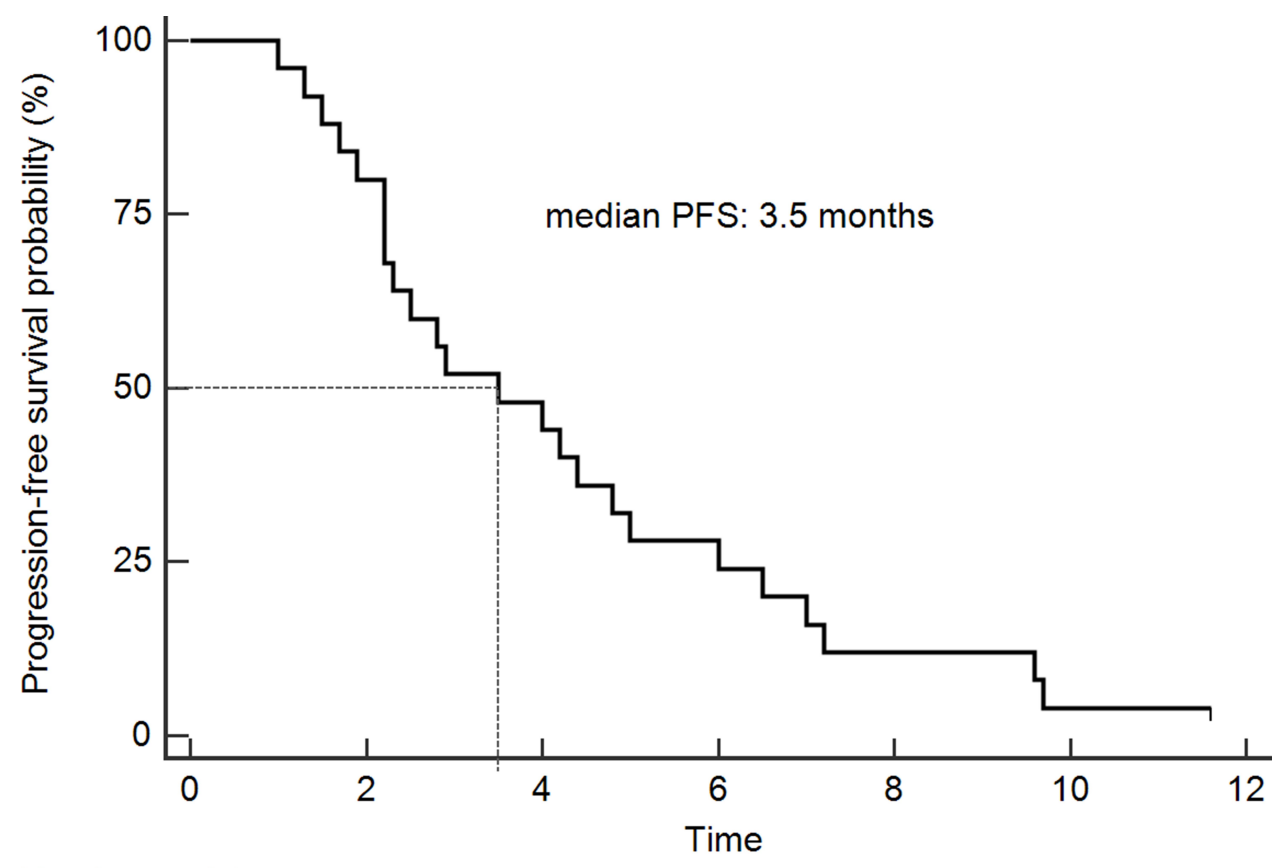

Number at risk

25

20

11

6

3

1

0

Figure 2 Kaplan-Meier curves of PFS for all 25 patients with HER2-positive non-breast advanced solid tumors.

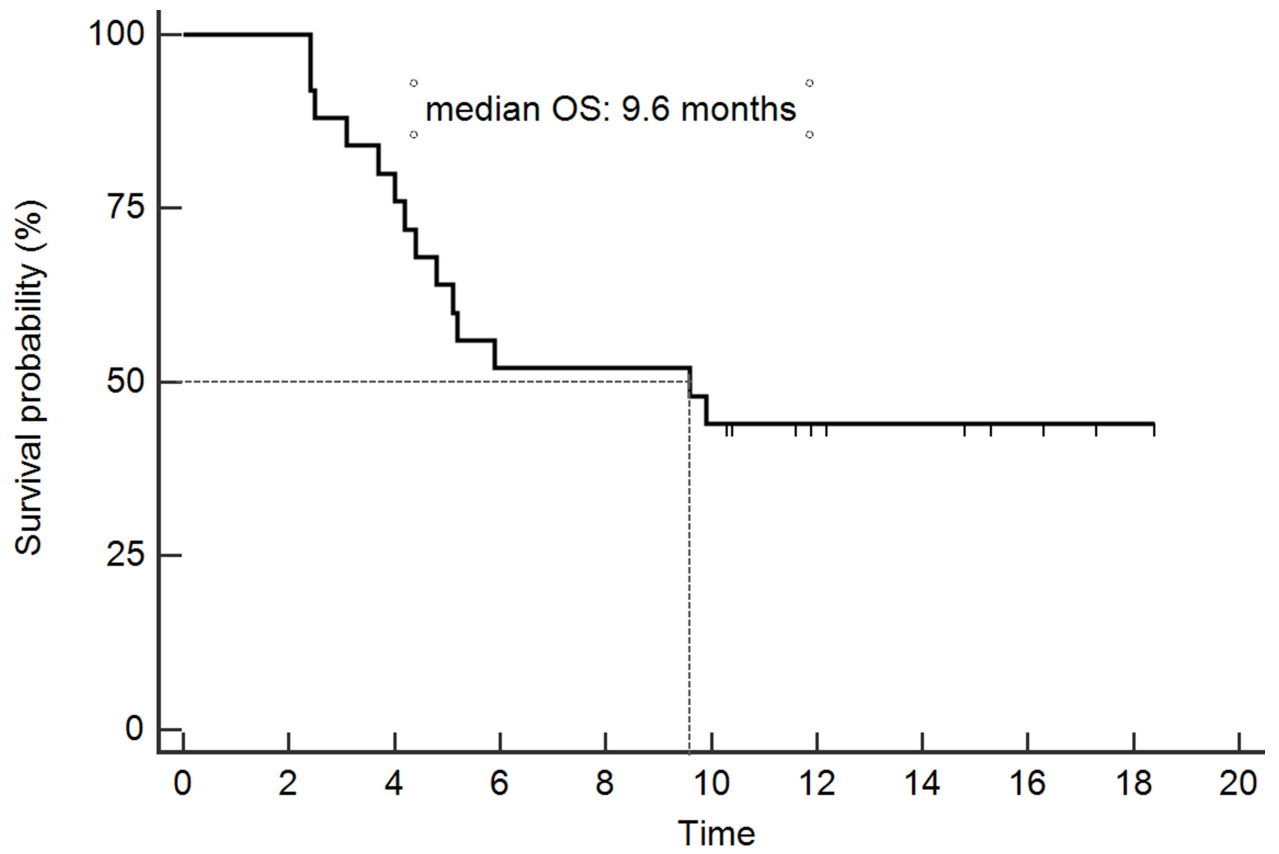

Number at risk

$\begin{array}{lllllllllll}25 & 25 & 19 & 13 & 13 & 11 & 7 & 6 & 4 & 2 & 0\end{array}$

Figure 3 Kaplan-Meier curve of OS in the overall study population $(n=25)$.

patients. In general, the ORR and DCR results indicated a promising anti-tumor effect of pyrotinib.

In addition, despite $20(80 \%)$ patients received pyrotinib-based therapy as a third or further line of treatment, our study showed an intriguing result of pyrotinib with a median PFS of 3.5 months and median OS of 9.6 months. The basket trials of T-DM1 did not obtain the results of median PFS and $\mathrm{OS}^{23}$ Additionally, 

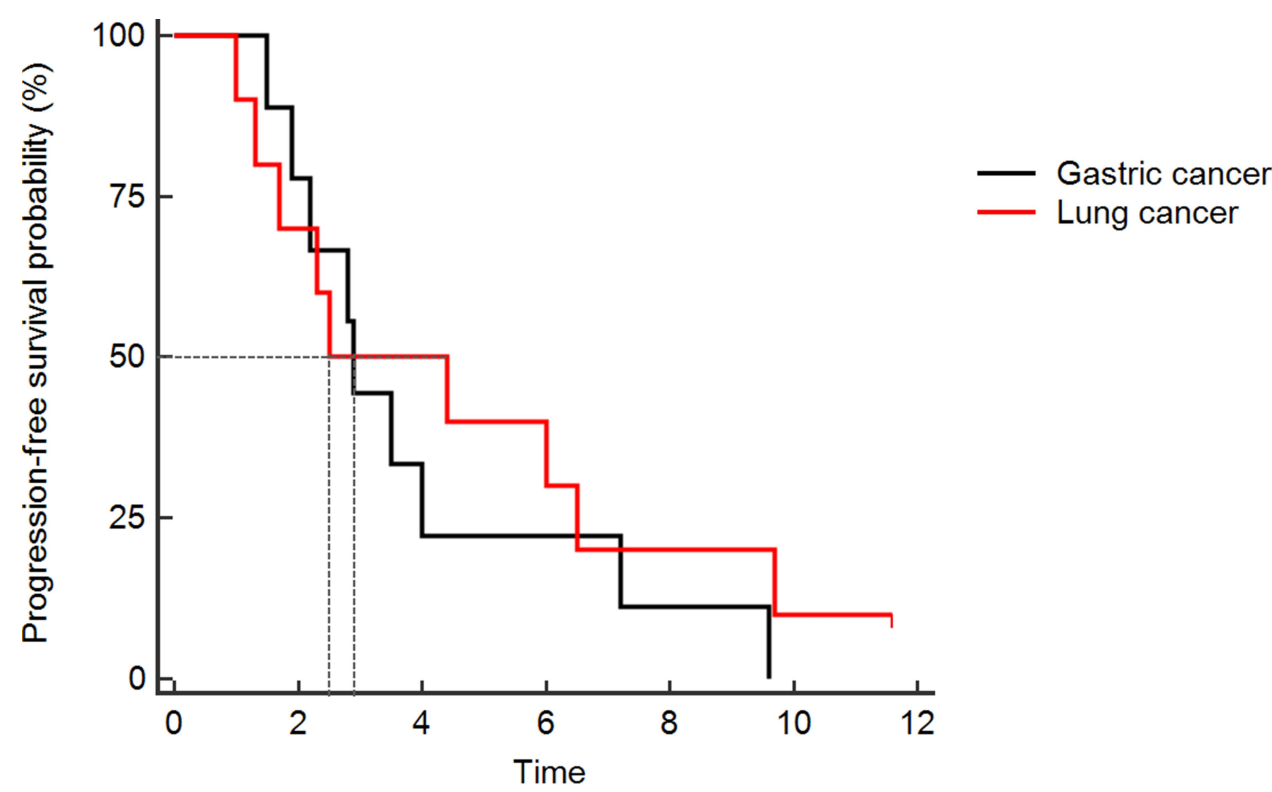

Number at risk

Gastric cancer

$\begin{array}{ccccccc}9 & 7 & 2 & 2 & 1 & 0 & 0 \\ \text { Lung cancer } & & & & & & \\ 10 & 7 & 5 & 3 & 2 & 1 & 0\end{array}$

Figure 4 Progression-free survival in 9 patients with gastric cancers and 10 patients with lung cancers.

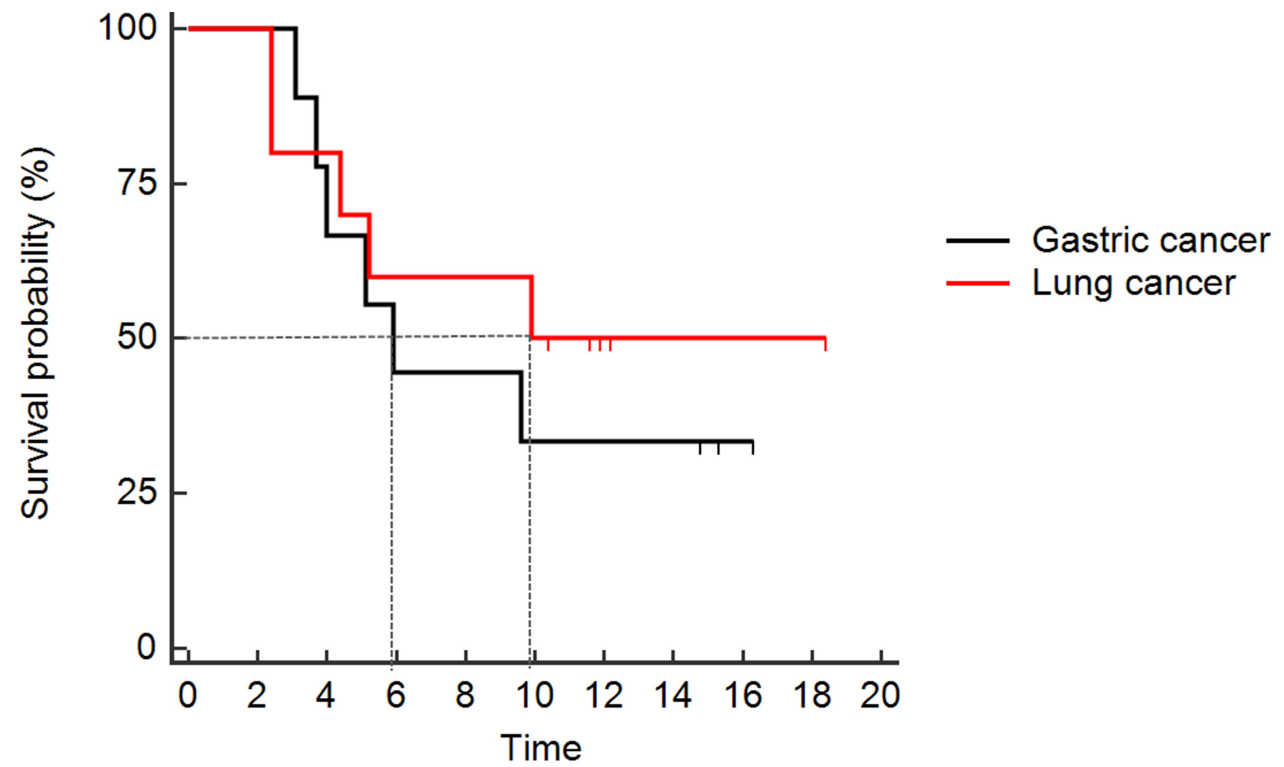

\section{Number at risk}

Gastric cancer

$\begin{array}{ccccccccccc}9 & 9 & 6 & 4 & 4 & 3 & 3 & 3 & 1 & 0 & 0 \\ \begin{array}{c}\text { Lung cancer } \\ 10\end{array} 10 & 8 & 6 & 6 & 5 & 2 & 1 & 1 & 1 & 0\end{array}$

Figure 5 Kaplan-Meier curve of OS for 9 patients with gastric cancers and 10 patients with lung cancers. 

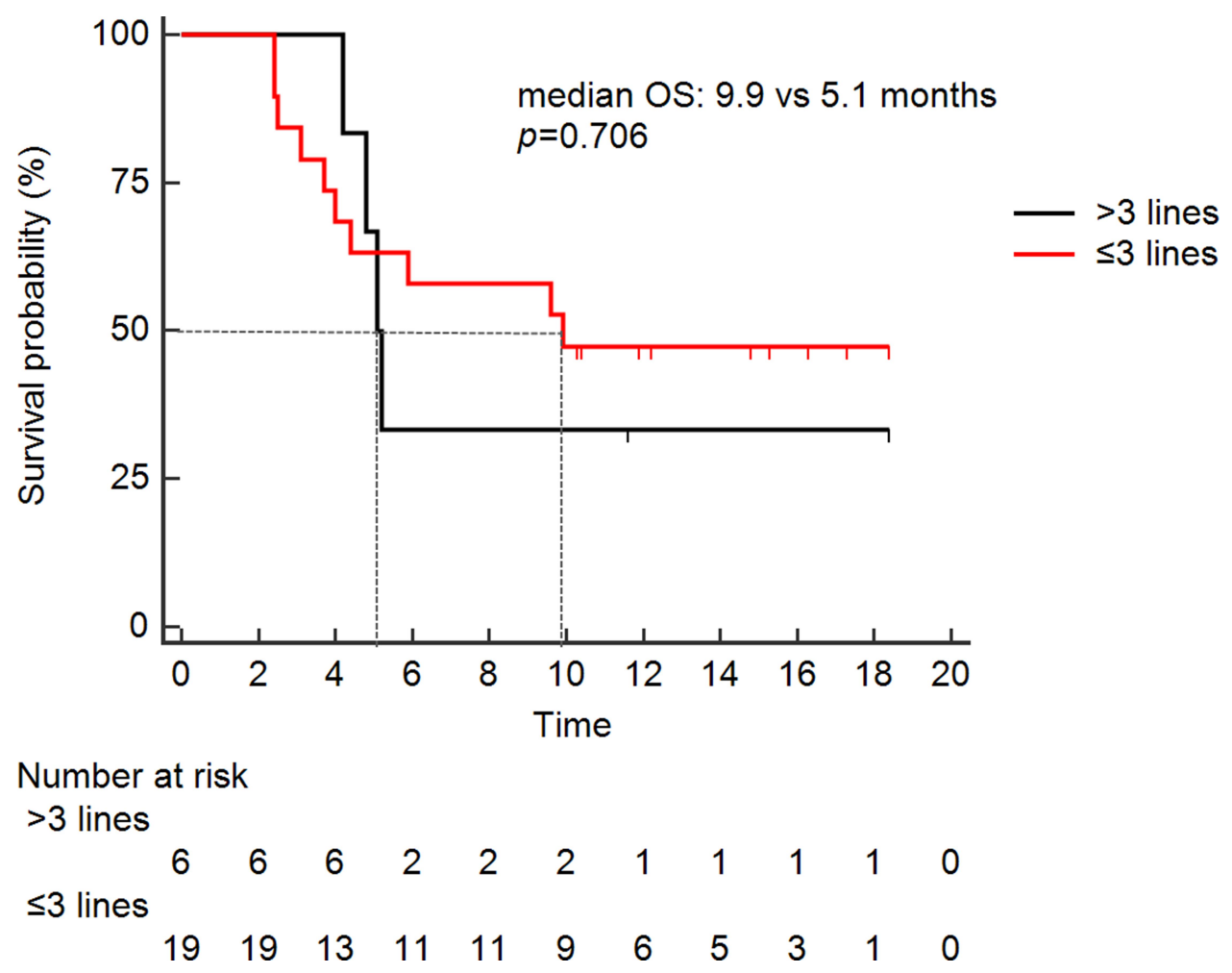

Figure 6 Survival analysis comparing $\leq 3$ lines and $>3$ lines of pyrotinib-containing treatments.

median PFS and OS were 2.9 months and 5.9 months in patients with gastric cancer, respectively. Eight (88.89\%) patients with gastric cancer were previously administered an anti-HER2 drug (trastuzumab). As trastuzumab is currently considered the standard first therapy in the treatment of HER2-positive gastric cancer, pyrotinib is an excellent choice for patients who progress after receiving trastuzumab. In addition, patients with lung cancer had a median PFS of 2.5 months and a median OS was 9.87 months. In a phase II trial, dacomitinib showed a median PFS of 3 months and a median OS of 9 months in patients with recurrent or de novo HER2mutant lung cancer. ${ }^{25}$ Our study showed clinical benefit with pyrotinib, especially in patients who had received

Table 5 Treatment-Related Adverse Events

\begin{tabular}{|l|l|l|}
\hline AE & All Grade, n (\%) & Grade $\geq 3, \mathbf{n}(\%)$ \\
\hline Diarrhea & $23(92 \%)$ & $5(20 \%)$ \\
Asthenia & $8(32 \%)$ & 0 \\
Hand-foot syndrome & $6(24 \%)$ & 0 \\
Vomiting & $2(8 \%)$ & \\
Stomatitis & $\mathrm{I}(4 \%)$ & \\
Anemia & $\mathrm{I}(4 \%)$ & \\
\hline
\end{tabular}

a third or further line of treatment. Moreover, patients receiving $\leq 3$ lines had a numerically higher median OS than those receiving $>3$ lines of treatment (5.1 vs 9.9). One of the potential reasons might be that pyrotinib is a novel, irreversible TKI with activity against EGFR/ HER 1, HER2, and HER4. In addition, pyrotinib significantly inhibited HER2 factor-driven tumor growth and HER2-mediated downstream signaling, and blocked tumor cells in the G1 phase of the cell cycle in mouse models of breast, lung, and ovarian cancer. ${ }^{13}$ These data showed that pyrotinib is an excellent choice for patients with HER2-positive advanced solid tumors excluding breast cancer.

The most common AE was diarrhea, but only 5 (20\%) patients reported grade 3 diarrhea. These results were similar to previous studies. ${ }^{26,27}$ Patients are instructed to start antidiarrhea treatment, treatment interruption, or dose reduction as early as possible. Early treatment after diarrhea could well control the incidence of diarrhea.

As this was a retrospective study, it is limited to include potential missing data and information bias. Moreover, the sample size of the study was small, and the results of the study need further confirmation in larger dedicated trials. 
In conclusion, pyrotinib-based therapy has promising efficacy for HER2-positive advanced solid tumors excluding breast cancer and toxicities could be well controlled. The study is just a pilot study motivating larger studies to elucidate the safety and efficacy of pyrotinib in non-breast solid tumors.

\section{Funding}

This study was supported by grants from suzhou talent training program (GSWS2019019).

\section{Disclosure}

The authors have no conflicts of interest to declare.

\section{References}

1. Slamon DJ, Godolphin W, Jones LA, et al. Studies of the HER-2/neu proto-oncogene in human breast and ovarian cancer. Science. 1989;244(4905):707-712. doi:10.1126/science.2470152

2. Eroglu Z, Tagawa T, Somlo G. Human epidermal growth factor receptor family-targeted therapies in the treatment of HER2-overexpressing breast cancer. Oncologist. 2014;19 (2):135-150. doi:10.1634/theoncologist.2013-0283

3. Giordano SH, Temin S, Kirshner JJ, et al. Systemic therapy for patients with advanced human epidermal growth factor receptor 2-positive breast cancer: American Society of Clinical Oncology clinical practice guideline. J Clin Oncol. 2014;32(19):2078-2099. doi:10.1200/jco.2013.54.0948

4. Swain SM, Baselga J, Kim SB, et al. Pertuzumab, trastuzumab, and docetaxel in HER2-positive metastatic breast cancer. $N$ Engl J Med. 2015;372(8):724-734. doi:10.1056/NEJMoa1413513

5. Verma S, Miles D, Gianni L, et al. Trastuzumab emtansine for HER2-positive advanced breast cancer. $N$ Engl J Med. 2012;367 (19):1783-1791. doi:10.1056/NEJMoa1209124

6. Cheng DT, Prasad M, Chekaluk Y, et al. Comprehensive detection of germline variants by MSK-IMPACT, a clinical diagnostic platform for solid tumor molecular oncology and concurrent cancer predisposition testing. BMC Med Genomics. 2017;10(1):33. doi:10.1186/ s12920-017-0271-4

7. Zehir A, Benayed R, Shah RH, Syed A, Middha S. Mutational landscape of metastatic cancer revealed from prospective clinical sequencing of 10,000 patients. Nat Med. 2017;23(6):703-713. doi:10.1038/ nm. 4333

8. Li BT, Shen R, Buonocore D, et al. Ado-trastuzumab emtansine for patients with HER2-mutant lung cancers: results from a Phase II basket trial. J Clin Oncol. 2018;36(24):2532-2537. doi:10.1200/ jco.2018.77.9777

9. Ter Veer E, van den Ende T. Continuation of trastuzumab beyond progression in HER2-positive advanced esophagogastric cancer: a meta-analysis. Acta Oncol. 2018;57(12):1599-1604. doi:10.1080/ 0284186x.2018.1503421

10. Fortman D, Issa R, Stanbery L, Albrethsen M, Nemunaitis J, Kasunic T. HER2-positive metastatic cervical cancer responsive to first and second-line treatment: a case report. Gynecol Oncol Rep. 2020;31:100520. doi:10.1016/j.gore.2019.100520
11. Li X, Yang C, Wan H, et al. Discovery and development of pyrotinib: a novel irreversible EGFR/HER2 dual tyrosine kinase inhibitor with favorable safety profiles for the treatment of breast cancer. Eur J Pharm Sci. 2017;110:51-61. doi:10.1016/j.ejps.2017.01.021

12. Zhu Y, Li L, Zhang G, et al. Metabolic characterization of pyrotinib in humans by ultra-performance liquid chromatography/quadrupole time-of-flight mass spectrometry. J Chromatogr B Analyt Technol Biomed Life Sci. 2016;1033-1034:117-127. doi:10.1016/j. jchromb.2016.08.009

13. Blair HA. Pyrotinib: first global approval. Drugs. 2018;78 (16):1751-1755. doi:10.1007/s40265-018-0997-0

14. Binghe X. Pyrotinib or lapatinib plus capecitabine for HER2+ metastatic breast cancer (PHOEBE): A randomized phase III trial. ASCO annual meeting; 2020.

15. NCCN clinical practice guidelines in oncology (NCCN Guidelines $\left.{ }^{\circledR}\right)$ : breast cancer, Version 5. 2020 - July 15, 2020.

16. Sekulic A, Migden MR, Lewis K, et al. Pivotal ERIVANCE basal cell carcinoma (BCC) study: 12-month update of efficacy and safety of vismodegib in advanced BCC. $J$ Am Acad Dermatol. 2015;72 (6):1021-1026.e1028. doi:10.1016/j.jaad.2015.03.021

17. Li BT, Ross DS, Aisner DL, et al. HER2 amplification and HER2 mutation are distinct molecular targets in lung cancers. $J$ Thorac Oncol. 2016;11(3):414-419. doi:10.1016/j.jtho.2015.10.025

18. Gerson JN, Skariah S, Denlinger CS, Astsaturov I. Perspectives of HER2-targeting in gastric and esophageal cancer. Expert Opin Investig Drugs. 2017;26(5):531-540. doi:10.1080/13543784.2017.1315406

19. Greally M, Kelly CM, Cercek A. HER2: an emerging target in colorectal cancer. Curr Probl Cancer. 2018;42(6):560-571. doi:10.1016/j.currproblcancer.2018.07.001

20. Hyman DM, Piha-Paul SA, Won H, et al. HER kinase inhibition in patients with HER2- and HER3-mutant cancers. Nature. 2018;554 (7691):189-194. doi:10.1038/nature25475

21. Hyman DM, Smyth LM, Donoghue MTA, et al. AKT inhibition in solid tumors with AKT1 mutations. J Clin Oncol. 2017;35 (20):2251-2259. doi:10.1200/jco.2017.73.0143

22. Hyman DM, Puzanov I, Subbiah V, et al. Vemurafenib in multiple nonmelanoma cancers with BRAF V600 mutations. $N$ Engl J Med. 2015;373(8):726-736. doi:10.1056/NEJMoa1502309

23. Jhaveri KL, Wang XV, Makker V, et al. Ado-trastuzumab emtansine (T-DM1) in patients with HER2-amplified tumors excluding breast and gastric/gastroesophageal junction (GEJ) adenocarcinomas: results from the NCI-MATCH trial (EAY131) subprotocol Q. Ann Oncol. 2019;30(11):1821-1830. doi:10.1093/annonc/mdz291

24. Chien AJ, Cockerill A, Fancourt C, et al. A phase 1b study of the Akt-inhibitor MK-2206 in combination with weekly paclitaxel and trastuzumab in patients with advanced HER2-amplified solid tumor malignancies. Breast Cancer Res Treat. 2016;155(3):521-530. doi:10.1007/s10549-016-3701-7

25. Kris MG, Camidge DR, Giaccone G, et al. Targeting HER2 aberrations as actionable drivers in lung cancers: phase II trial of the pan-HER tyrosine kinase inhibitor dacomitinib in patients with HER2-mutant or amplified tumors. Ann Oncol. 2015;26 (7):1421-1427. doi:10.1093/annonc/mdv186

26. Ma F, Ouyang Q, Li W, et al. Pyrotinib or lapatinib combined with capecitabine in HER2-Positive metastatic breast cancer with prior taxanes, anthracyclines, and/or trastuzumab: a randomized, Phase II study. J Clin Oncol. 2019;37(29):2610-2619. doi:10.1200/jco.19.00108

27. Li Q, Guan X, Chen S, et al. Safety, efficacy, and biomarker analysis of pyrotinib in combination with capecitabine in HER2-positive metastatic breast cancer patients: a Phase I clinical trial. Clin Cancer Res. 2019;25(17):5212-5220. doi:10.1158/1078-0432.ccr-18-4173 


\section{Publish your work in this journal}

Cancer Management and Research is an international, peer-reviewed open access journal focusing on cancer research and the optimal use of preventative and integrated treatment interventions to achieve improved outcomes, enhanced survival and quality of life for the cancer patient.
The manuscript management system is completely online and includes a very quick and fair peer-review system, which is all easy to use. Visit http://www.dovepress.com/testimonials.php to read real quotes from published authors. 